\title{
Acceptance of COVID-19 Vaccination: Cross-Sectional Survey of Adults with Nonadherence to Physician- Recommended Statin Medications
}

\author{
Derjung M. Tarn, MD, PhD ${ }^{1^{*}}$, Kevin Shih, $P h D^{1}$ and Janice B. Schwartz, $M D^{2}$ \\ ${ }^{1}$ Department of Family Medicine, David Geffen School of Medicine at UCLA, University of California, USA \\ ${ }^{2}$ Division of Geriatrics, Department of Medicine and Division of Clinical Pharmacology, Departments of Medicine and \\ Bioengineering and Therapeutic Sciences, University of California, USA
}

\section{Background}

COVID-19 vaccine uptake is a critical public health challenge. Suboptimal COVID-19 vaccination rates in the United States have highlighted the need to understand drivers of resistance to vaccination, which one could consider nonadherence to a preventive therapy. The literature shows that physicians strongly influence patient decisions about vaccination [1], but knowledge is lacking regarding the relationship of patient acceptance of vaccinations and their acceptance of other physician recommendations regarding preventive treatments. This study investigated willingness to receive the COVID-19 vaccine in patients who are nonadherent to medications for prevention of cardiovascular risk reduction.

\section{Methods}

This study invited participants from a University of California, Los Angeles (UCLA) survey conducted from MayAugust 2019, prior to the onset of the COVID-19 pandemic [2]. The original study cohort was identified and recruited through UCLA large-scale electronic health record data extractions and online advertisements on Craigslist.com. Eligibility criteria included adults aged 18 and older who were prescribed an HMG-CoA reductase inhibitor medication, but self-reported nonadherence to the medication. For this study, we emailed Qualtrics survey invitations to original study participants who agreed to be contacted for future studies. Nonrespondents received 2 follow-up emails, 1 week apart. Surveys were conducted from January 6, 2021, to February 5,2021 . We asked respondents whether they received or intended to receive the COVID-19 vaccine, and about past administration and future plans for influenza vaccination. Responses were linked to previously collected data on respondent demographics and health conditions. Descriptive statistics were calculated, and tests of significance were performed using Fisher's exact and Wilcoxon-Mann-Whitney tests. The UCLA Institutional Review Board approved the study protocol.

\section{Results}

Of 211 emailed invitations, 42 were undeliverable, 6 invitees opted-out, and 1 response was incomplete. Fiftytwo respondents completed the survey $(31 \%$ of delivered invitations). Respondents were mostly non-Hispanic white $(83 \%)$ and female (54\%), and most had completed college or graduate school (69\%). Forty percent reported having cardiovascular disease. Only 7 of the originally nonadherent (13\%) reported current statin use.

Most survey respondents (82\%) were either planning on or had received the COVID-19 vaccine. Those who were accepting of the vaccine were more likely than those not planning on getting it to be male $(p<0.01)$, non-Hispanic white $(p<0.01)$, and to have diabetes $(p=0.03)$ or cardiovascular disease $(p<0.01)$ (Table 1$)$.

We found individuals accepting of the COVID-19 vaccine were significantly more likely to have received or to plan on getting the influenza vaccine, compared to those who were unaccepting of the vaccine ( $93 \%$ versus $22 \%$; $p<0.001$ ). We found no differences in COVID-19 vaccine acceptance based on prior medication adherence or influenza vaccination status.

\section{Discussion}

We found the majority of individuals who were previously nonadherent to physician recommendations to take statin

*Corresponding author: Derjung Mimi Tarn, MD, PhD, Department of Family Medicine, David Geffen School of Medicine at UCLA, 10880 Wilshire Blvd, Suite 1800, Los Angeles, CA 90024, USA

Accepted: January 29, 2022

Published online: January 31, 2022

Citation: Tarn DM, Shih K, Schwartz JB (2022) Acceptance of COVID-19 Vaccination: Cross-Sectional Survey of Adults with Nonadherence to Physician-Recommended Statin Medications. Arch Fam Med Gen Pract 6(1):178-180 
Citation: Tarn DM, Shih K, Schwartz JB (2022) Acceptance of COVID-19 Vaccination: Cross-Sectional Survey of Adults with Nonadherence to Physician-Recommended Statin Medications. Arch Fam Med Gen Pract 6(1):178-180

Table 1: Participant characteristics, overall and by acceptance of COVID-19 vaccination.

\begin{tabular}{|c|c|c|c|c|}
\hline Characteristic & All respondents & $\begin{array}{l}\text { Planning on or received } \\
\text { COVID-19 vaccine }\end{array}$ & $\begin{array}{l}\text { Not planning on } \\
\text { COVID-19 vaccine }\end{array}$ & p-value \\
\hline Total respondents, n (\%) & $52(100)$ & $43(83)$ & $9(17)$ & \\
\hline Age, mean (SD; range) & $53.4(10.2 ; 33-75)$ & $52.7(10.5 ; 33-75)$ & $56.7(8.4 ; 45-72)$ & 0.16 \\
\hline \multicolumn{4}{|l|}{ Gender, n (\%) } & \multirow{3}{*}{0.002} \\
\hline Female & $28(54)$ & $19(44)$ & $9(100)$ & \\
\hline Male & $24(46)$ & $24(56)$ & 0 & \\
\hline \multicolumn{4}{|l|}{ Race/ethnicity, n (\%) } & \multirow{3}{*}{0.005} \\
\hline Non-Hispanic White & $43(83)$ & $39(91)$ & $4(44)$ & \\
\hline Other & $9(17)$ & $4(9)$ & $5(56)$ & \\
\hline \multicolumn{4}{|l|}{ Education, n (\%) } & \multirow{5}{*}{0.14} \\
\hline High school or less & $6(12)$ & $3(7)$ & $3(33)$ & \\
\hline Some college & $10(19)$ & $8(19)$ & $2(22)$ & \\
\hline College graduate & $25(48)$ & $22(51)$ & $3(33)$ & \\
\hline Graduate school & $11(21)$ & $10(23)$ & $1(11)$ & \\
\hline Cardiovascular disease, ${ }^{*} \mathrm{n}(\%)$ & $21(40)$ & $21(49)$ & $0(0)$ & 0.007 \\
\hline Diabetes, n (\%) & $24(46)$ & $23(54)$ & $1(11)$ & 0.03 \\
\hline Hypertension, n (\%) & $31(60)$ & $25(58)$ & $6(67)$ & 0.72 \\
\hline \#of health conditions, ${ }^{* *}$ mean (SD; range) & $1.48(1.06 ; 0-3)$ & $1.63(1.07 ; 0-3)$ & $0.78(0.67 ; 0-2)$ & 0.03 \\
\hline \#of prescription medications, mean (SD; range) & $2.10(1.49 ; 0-6)$ & $2.09(1.41 ; 0-6)$ & $2.11(1.90 ; 0-5)$ & 0.84 \\
\hline \multicolumn{4}{|l|}{ Smoking, n (\%) } & \multirow{3}{*}{0.44} \\
\hline Yes & $3(6)$ & $2(5)$ & $1(11)$ & \\
\hline No & $49(94)$ & $41(95)$ & $8(89)$ & \\
\hline \multicolumn{4}{|l|}{$\begin{array}{l}\text { Received influenza vaccine during 2019-2020 flu } \\
\text { season }\end{array}$} & \multirow{3}{*}{0.14} \\
\hline Yes & $18(35)$ & $17(40)$ & $1(11)$ & \\
\hline No & $34(65)$ & $26(60)$ & $8(89)$ & \\
\hline \multicolumn{4}{|l|}{$\begin{array}{l}\text { Influenza vaccination during 2020-2021 flu season, } \\
\text { n (\%) }\end{array}$} & \multirow{4}{*}{$<0.001$} \\
\hline Already received & $17(33)$ & $15(35)$ & $2(22)$ & \\
\hline Plan on getting & $25(48)$ & $25(58)$ & $0(0)$ & \\
\hline Not planning on getting & $10(19)$ & $3(7)$ & $7(78)$ & \\
\hline \multicolumn{4}{|l|}{ Statin nonadherence, $\mathrm{n}(\%)$} & \multirow{3}{*}{1} \\
\hline Primary & $47(90)$ & $39(91)$ & $8(89)$ & \\
\hline Secondary & $5(10)$ & $4(9)$ & $1(11)$ & \\
\hline \multicolumn{4}{|l|}{ Currently taking a statin, $\mathrm{n}(\%)$} & \multirow{3}{*}{0.59} \\
\hline Yes & $7(14)$ & $5(12)$ & $2(22)$ & \\
\hline No & $45(86)$ & $38(88)$ & $7(78)$ & \\
\hline \multicolumn{4}{|l|}{ Original recruitment method, $\mathrm{n}(\%)$} & \multirow{3}{*}{0.14} \\
\hline Online classified advertisements & $26(50)$ & $24(56)$ & $2(22)$ & \\
\hline Academic medical centers & $26(50)$ & $19(44)$ & $7(78)$ & \\
\hline
\end{tabular}

"Cardiovascular disease: Myocardial infarction, stroke or transient ischemic attack, angina; ${ }^{* *}$ Patients were asked if they had: Cardiovascular disease, diabetes, hypertension, or chronic kidney disease; maximum "health conditions: 4. 
Citation: Tarn DM, Shih K, Schwartz JB (2022) Acceptance of COVID-19 Vaccination: Cross-Sectional Survey of Adults with Nonadherence to Physician-Recommended Statin Medications. Arch Fam Med Gen Pract 6(1):178-180

medications were accepting of the COVID-19 vaccine. In the United States, the COVID-19 vaccine has elicited attitudes that are similar, if not even more negative than those concerning statins [3]. We hypothesized that people expressing resistance to proven and recommended preventive strategies for cardiovascular disease [4] might be skeptical of COVID-19 vaccines. The obvious conclusion from our study is that perceptions regarding one preventive intervention do not predict responses to a different preventive intervention. There are many possible explanations for this discordance in acceptance or rejection of disease prevention modalities. These may include constant reminders of the immediate mortality and morbidity risks of COVID-19 infections in unvaccinated people, compared to only risk estimates for cardiovascular events far in the future that do not focus on death or major disability. Other explanations include the transient nature of any adverse effects from vaccines versus the need for daily consumption of a medication, concern for transmitting infections to high-risk loved ones, free COVID-19 vaccines regardless of insurance, and desires to have social contacts advised only for vaccinated individuals, among others. Perhaps encouragingly, it appears that broader acceptance of COVID vaccination may result in greater acceptance of influenza vaccination.

Study limitations include participation from mostly nonHispanic, white respondents with higher education levels, and from those with medical conditions that put them at higher risk for adverse outcomes from COVID-19 infections [5].

Our findings suggest a role for strong messaging regarding the serious risks of coinfection [6] when promoting the COVID-19 and influenza vaccines. In addition, the data suggest that nonadherence to one medication for primary prevention may not predict acceptance or rejection of preventive measures for other diseases. Finally, the data suggest that patients are more receptive to preventive measures when faced with the possibility of imminent serious illness or death. Future studies could investigate the most effective ways to communicate risk factors, as the need for preventive measures such as COVID-19 and influenza vaccines will continue in the future.

\section{Acknowledgements}

Funders: This project was supported in part by NIH/NIA grant 1R21AG055832, and the National Center for Advancing Translational Sciences, National Institutes of Health (UCLA CTSI UL1TR001881). The contents are solely the responsibility of the authors and do not necessarily represent the official views of the $\mathrm{NIH}$.

\section{Conflicts of Interest}

Drs. Tarn and Schwartz have been funded by the BMS/ Pfizer Alliance ARISTA-USA for unrelated research studies.

\section{References}

1. Edwards KM, Hackell JM (2016) Countering vaccine hesitancy, The committee on infectious diseases and the committee on practice ambulatory medicine. Pediatrics 138: e20162146.

2. Tarn DM, Pletcher MJ, Tosqui R, et al. (2021) Primary nonadherence to statin medications: Survey of patient perspectives. Preventive Medicine Reports 22: 101357.

3. Al-Amer R, Maneze D, Everett B, et al. (2021) COVID-19 vaccination intention in the first year of the pandemic: $A$ systematic review. J Clin Nurs 31: 62-86.

4. Grundy SM, Stone NJ, Bailey AL, et al. (2019) 2018 AHA/ACC/ AACVPR/AAPA/ABC/ACPM/ADA/AGS/APhA/ASPC/NLA/PCNA Guideline on the Management of Blood Cholesterol: A report of the American college of cardiology/american heart association task force on clinical practice guidelines. Circulation 139: e1082-e1143.

5. Xu J, Xiao W, Liang X, et al. (2021) A meta-analysis on the risk factors adjusted association between cardiovascular disease and COVID-19 severity. BMC Public Health 21: 1533.

6. Stowe J, Tessier E, Zhao H, et al. (2021) Interactions between SARS-CoV-2 and influenza, and the impact of coinfection on disease severity: A test-negative design. Int J Epidemiol 50: 1124-1133.

DOI: $10.36959 / 577 / 503$

Copyright: (C) 2022 Tarn DM, et al. This is an open-access article distributed under the terms of the Creative Commons Attribution License, which permits unrestricted use, distribution, and reproduction in any medium, provided the original author and source are credited. 